\title{
Using Continuations to Account for Plural Quantification and Anaphora Binding
}

Anca Dinu

\begin{abstract}
We give in this paper an explicit formal account of plural semantics in the framework of continuation semantics introduced in [1] and extended in [4]. We deal with aspects of plural dynamic semantics such as plural quantification, plural anaphora, conjunction and disjunction, distributivity and maximality conditions. Those phenomena need no extra stipulations to be accounted for in this framework, because continuation semantics provides a unified account of scopetaking.
\end{abstract}

Index Terms-Discourse semantics, continuations, plural anaphora, plural quantifiers' scope.

\section{INTRODUCTION}

$\mathrm{T}$ HE formal study of plurals is a major and complex undertaking. It should address plural quantification, plural anaphora, conjunction and disjunction, distributivity, maximality, among other problems. Ideally, plural linguistic phenomena should be parallel to their singular counterpart. Unfortunately, it is not so: plurality introduces a number of complications not present in the analysis of singular. In this paper we will only give some directions for further study of plural semantics (our analysis is far from being exhaustive), with emphasis on plural anaphora, in the framework of continuation semantics.

The programming language concept of continuations was successfully used by Barker and Shan in a series of articles to analyze intra-sentential linguistic phenomena such as focus fronting, donkey anaphora, presuppositions, crossover or superiority [2, 3, 19, 18, 1]. Moreover, [9] proposed an elegant discourse semantics based on continuations. Continuations are a standard tool in computer science, used to control side effects of computation (such as evaluation order, print or passing values). The basic idea of continuizing a grammar is to provide subexpressions with direct access to their own continuations (future context), so subexpressions are modified to take a continuation as an argument. For instance, if we take the local context to be restricted to the sentence, when computing the meaning of the sentence John saw Mary, the default future of the value denoted by the subject is that it is destined to have the property of seeing Mary predicated of it. In symbols, the continuation of the subject denotation $\boldsymbol{j}$ is the function $\lambda x$ saw $m x$. Similarly, the default future of the object denotation $\boldsymbol{m}$ is the property of being seen by John, the

Manuscript received on November 1, 2011, manuscript accepted on December 9, 2011.

The author is with the Centre for Computational Linguistics, University of Bucharest, Romania (e-mail: anca_d_dinu@yahoo.com). function $\lambda y$ s saw $y j$; the continuation of the transitive verb denotation saw is the function $\lambda R . R \boldsymbol{m} \mathbf{j}$; and the continuation of the VP saw Mary is the function $\lambda P . P \boldsymbol{j}$. This simple example illustrates two important aspects of continuations: every meaningful subexpression has a continuation; and the continuation of an expression is always relative to some larger expression containing it. Thus, when John occurs in the sentence John left yesterday, its continuation is the property 7x. yesterday left $\mathrm{x}$; when it occurs in Mary thought John left, its continuation is the property $\lambda x$, thought (left $x$ ) $\boldsymbol{m}$ and so on.

In what follows we will give a short survey of the continuations semantic framework in which we will analyze plurals.

One of the main challenges of interpreting a discourse (giving it a compositional semantics) is interpreting crosssentential anaphora. Assigning a first order logical representation to a discourse like $A$ man came. He whistled is problematic. How can we get from the two first order representations in (1) and (2) the representation in (3), i.e. obtaining the bound variable whistled(x) in (3) from the free one in (1)?

$$
\begin{aligned}
& \exists x \cdot(\operatorname{man}(x) \wedge \operatorname{came}(x)) \\
& \text { whistled }(x) \\
& \exists x .(\operatorname{man}(x) \wedge \operatorname{came}(x)) \wedge \text { whistled }(x))
\end{aligned}
$$

Various dynamic semantic theories that handle this were proposed, for instance in Discourse Representation Theory [12], File Change Semantics [10], Dynamic Montague Grammar [7], Dynamic Predicate Logic [8], Jacobson's variable free semantics [11]. All these theories have also something to say about the complex semantics of plurals (for instance about the plural version of the above discourse: Some man came. They whistled), complications included. We have chosen to treat plural semantics in the framework of continuation semantics because it provides a unified account of scope-taking (quantification and binding employ the same mechanism), and thus an elegant treatment of anaphora (be it singular or plural).

We will use Barker's tower notation for a given expression, which consists of three levels: the top level specifies the syntactic category of the expression coached in categorical grammar (the categories act either as functions or as attributes), the middle level is the expression itself and the bottom level is the semantic value. 


\section{syntactic category \\ expression \\ semantic value}

The syntactic categories are written $\frac{\mathrm{C}] \mathrm{B}}{\mathrm{A}}$, where A, B and C can be any categories. We read it counter clockwise: the expression functions as category A in local context, takes scope at an expression of category B to form an expression of category $\mathrm{C}$.

The semantic value $\lambda k \cdot f[k(x)]$ is equivalently written vertically as $\frac{f[1]}{x}$ omitting the future context (continuation) $k$. Here, $x$ can be any expression, and $f[]$ can be any expression with a gap [ ]. Free variables in $x$ can be bound by binders in $f$ [ ]. This notational convention is meant to make easier (more visual) then in linear notation the combination process of two expressions. [1] gives the two possible modes of combination:

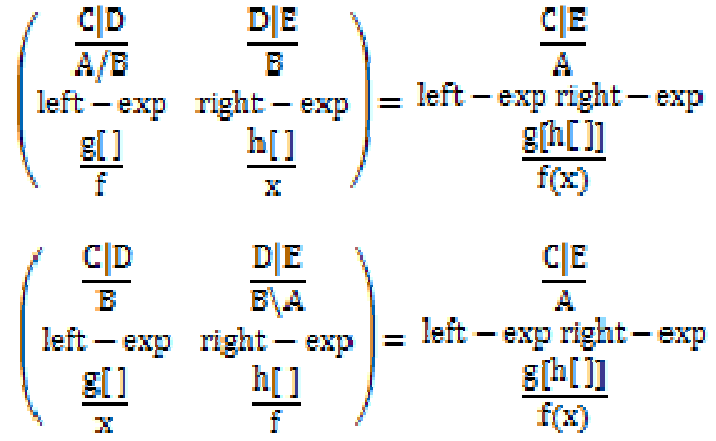

Below the horizontal lines, combination proceeds simply as in combinatory categorical grammar: in the syntax, B combines with $A / B$ or $B \backslash A$ to form $A$; in the semantics, $\mathrm{x}$ combines with $\mathrm{f}$ to form $f(x)$. Above the lines is where the combination machinery for continuations is employed. The syntax combines the two pairs of categories by cancellation: the $D$ on the left cancels with the $D$ on the right. The semantics combines the two expressions with gaps by composition: we plug $h[]$ to the right into the gap of $g[]$ to the left, to form $g[h[]]$. The expression with a gap on the left, $g[]$, always surrounds the expression with a gap on the right, $h[]$, no matter which side supplies the function or the argument below the lines. This fact expresses the generalization that the default order of semantic evaluation is left-to-right.

When there is no quantification or anaphora involved, a simple sentence like John came is derived as follows:

$$
\left(\begin{array}{cc}
D P & D P \backslash S \\
J \text { ohn } & \text { came } \\
j & \text { came }
\end{array}\right)=\begin{gathered}
S \\
\text { John came } \\
\text { camej }
\end{gathered}
$$

In the syntactic layer, as usual in categorical grammar, the category under slash (here DP) cancels with the category of the argument expression; the semantics is function application.

Quantificational expressions have extra layers on top of their syntactic category and on top of their semantic value, making essential use of the powerful mechanism of continuations in ways proper names or definite descriptions do not. For example, below is the derivation for $A$ man came:

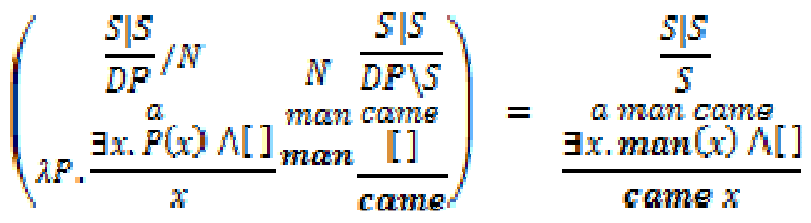

Comparing the analysis above of John came with that of $A$ man came reveals that came has been given two distinct values. The first, simpler value is the basic lexical entry, the more complex value being derived through the standard typeshifter Lift, proposed by [15] and many others:

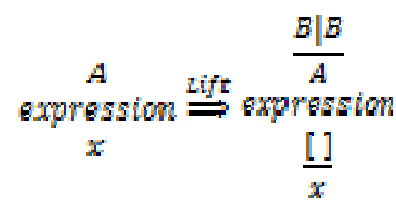

Syntactically, Lift adds a layer with arbitrary (but matching) syntactic categories. Semantically, it adds a layer with empty brackets. In linear notation we have: $x \stackrel{\text { Lift }}{\Longrightarrow} \lambda k, k(x)$.

To derive the syntactic category and a semantic value with no horizontal line, [1] introduce the type-shifter Lower. In general, for any category $A$, any value $x$, and any semantic expression $f[]$ with a gap, the following type-shifter is available.

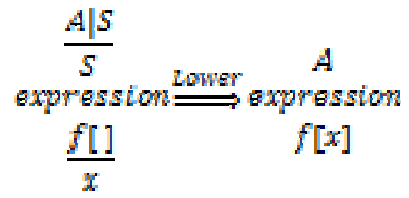

Syntactically, Lower cancels an $S$ above the line to the right with an $S$ below the line. Semantically, Lower collapses a twolevel meaning into a single level by plugging the value $x$ below the line into the gap [ ] in the expression $f$ [ ] above the line. Lower is equivalent to identity function application.

The third and the last type shifter we need is the one that treats binding. Binding is a term used both in logics and in linguistics with analog (but not identical) meaning. In logics, a variable is said to be bound by an operator (as the universal or existential operators) if the variable is inside the scope of the operator. If a variable is not in the scope of any operator then the variable is said to be free. In linguistics, a binder may be a constituent such as a proper name (John), an indefinite common noun $(a$ book), an event or a situation. Anaphoric expressions such as pronouns (he, she, it, him, himself, etc), definite common nouns (the book, the book that John read), demonstrative pronouns (like this, that), etc act as variables that take the value of (are bind by) a previous binder. We adopt the idea (in line with [1]) that the mechanism of binding is the same as the mechanism of scope taking.

In order to give a proper account of anaphoric relations in discourse, we need to formulate an explicit semantics for both the binder and the anaphoric expressions to be bound. Any DP may act as a binder, as the Bind rule from [1] explicitly states:

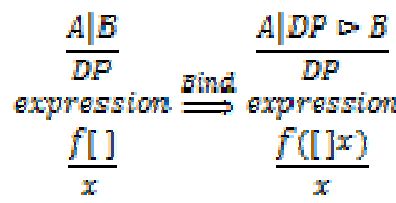


At the syntactic level, Bind says that an expression that functions in local context as a DP may look to the right to bind an anaphoric expression (encoded by the sign $\approx$ ). At the semantic level, the expression transmits the value of the variable $x$. In linear notation, the semantic part of Bind looks like: $\lambda k, \mathrm{f}[\mathrm{k}(\mathrm{x})] \stackrel{\text { aind }}{\Longrightarrow} \lambda \mathrm{k}, \mathrm{f}([\mathrm{k}(\mathrm{x})] \mathrm{x})$

As for the elements that may be bound, [1] give for instance the following lexical entry for singular pronoun $h e$ :

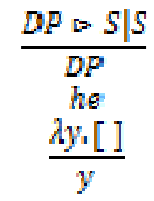

Distinct scope-taking levels correspond to different binders, layers playing the role of indices: a binder and the pronoun it binds must take effect at the same layer in the compositional tower. A superior level takes scope at inferior levels and left expressions take scope at right expressions, to account for left-to-right natural language order of processing.

In order to account for discourse phenomena, [4] gives the semantics of the dot as a function that take two sentence denotations and returns a sentence denotation (their conjunction):

$$
\begin{gathered}
S \backslash(S / S) \\
\lambda p \lambda q \cdot p / q
\end{gathered}
$$

\section{Plural Quantificational Determiners}

There is a vast literature on representing plurals. We will only refer to two of the most influential existing approaches: the proposals of Scha [17] and of Link [13]. The most well-known and largely accepted view of natural language quantification is the generalized quantifiers view (Montague [14] and many others). The generalized quantifier type $<<<e, t>, t>, t>$ is exactly the type of quantificational determiners in continuation-based semantics. This is by no means a coincidence, generalized quantifiers approach only continuizes the noun phrase meanings rather than continuizing uniformly throughout the grammar as it is done in continuation-based semantics.

A tradition going back at least to Evans [5] says that the scope of all quantifiers is clause bounded. An E-type (or donkey) pronoun is a pronoun that lies outside the restrictor of a quantifier or outside the antecedent of a conditional, yet covaries with some quantificational element inside it, usually an indefinite. Here there are some of the famous donkey sentences examples:

\section{If a farmer owns a donkey, he beats it.}

Every farmer who owns a donkey beats it.

Evans made it standard to assume that the indefinite $a$ donkey cannot take scope over the pronoun it, and therefore cannot bind it, at least not in the ordinary sense of binding. To the contrary, as [1] put forward, the relationship between $a$ donkey and it in the above examples seems like binding because it is just binding: the scope of the indefinite a donkey stretches over the consequent of the conditional and binds the pronoun $i t$. In what follows, manipulating the scope of plural quantificational determiners in a similar liberal manner (in which we do not restrict it to clause boundaries) will allow us to account for a wide range of linguistic data involving plural anaphora binding.

Plural quantificational determiners take as arguments plural common nouns. Among other constructions such as coordinated DPs or singular DPs, they introduce plural referring variables. From a technical point of view, a plural referring variable notated with upper letters $(X, Y, Z, .$.$) is a set$ of entities.

We will give lexical entries for the plural quantificational determiners some (not to be confused with its singular counterpart), all and most, in the continuation semantics framework. Some and most need special care when the plural variable they introduce binds some subsequent anaphora, due to the so-called maximality constraint. While Some kids came. (with no other subsequent anaphora that refers to the kids that came) means that there is a set of any cardinal of kids that came, the discourse Some kids came. They played. means that there is a maximal set of kids who came and that maximal set played. So, there is a maximality operator that blocks further transmission of arbitrary sets, much like the negation blocks transmission of values of indefinites in direct object position to subsequent anaphora. The two uses of some have different truth-conditions. When some is used in the first, weak sense, we take it to have the following lexical entry:

$$
\begin{aligned}
& \frac{S \mid S}{D P^{p !}} / N^{p l} \\
& A P, \frac{\exists X \cdot(|X| \geq 1 \wedge P(X) \wedge[]}{X}
\end{aligned}
$$

Then, we have to force the scope closing of the variable $X$ in the usual way by applying Lower, in order to forbid it to bind subsequent anaphora (transmit a non-maximal value).

When used in the second, maximal sense, that exports a maximal set to bind a subsequent anaphora (such as they), we take some to have the alternative lexical entry:

$$
\begin{aligned}
& \frac{S \mid D P^{p l}=S}{\frac{S \mid S}{D P^{p /} / N^{p l}}} \\
& \operatorname{son} \theta \\
& \exists X .|X| \geq 1 \wedge[] X \\
& \lambda P, \frac{\left.X=\operatorname{argmax} \mid f Y_{P} P(Y) \cap[]\right] \mid}{Y}
\end{aligned}
$$

Note that we could have not used in this case the regular Bind rule, because of the intervening level that contains argmax. This level blocs the transmission of variable $Y$ and only lets the maximal variable $X$ to bind subsequent anaphora.

For the same reasons, we similarly treat the quantificational determiner most, for which we propose the following two alternative lexical entries, one for the weak sense, and one for strong (maximal) sense, respectively: 


$$
\begin{aligned}
& \frac{\frac{3 / 3}{D P^{p I}}}{N^{p L}} \\
& \lambda P, \frac{\exists X, P(X) \cap[] \cap 2|X| \geq|[x, P x\}|}{X} \\
& \frac{S \mid D P^{p l}-S}{\frac{S \mid S}{D P^{P l} / N^{p l}}} \\
& \exists X, 2|X| \geq \| x F_{x} \mid \wedge[] X \\
& \lambda P \cdot \frac{X=\operatorname{argmax}|[Y, P(Y) \wedge[]\}|}{Y}
\end{aligned}
$$

For the quantificational determiner all, the maximality condition has limited scope only over the restrictor $P$, thus we can give it a single lexical entry:

$$
\begin{aligned}
& \frac{S \| s}{D p^{2}} / N^{p l} \\
& \frac{\left.\left.\left.\exists X .\left(X=\operatorname{argmax} \mid\left[V_{1} P(Y)\right]\right)\right][]\right] \mid\right)}{X}
\end{aligned}
$$

It has been argued that all is not a quantificational determiner proper, but more like a modifier. It may be for that reason that it behaves differently compared to genuine quantificational determiners.

We turn now to the problem of compositionally obtaining the meaning of bare plurals. Bare plurals are plurals without overt article. Sentences with bare plurals can have either existential readings (John gave Mary flowers), or universal (generic) readings (Flowers are beautiful). We propose that the existential reading is accounted for by a silent quantificational determiner that has the same semantics as some (i.e. both weak and maximal senses). The universal reading is accounted for by a similar silent quantificational determiner, having the semantics of all:

$$
\begin{aligned}
& \frac{S \| S}{D P^{21}} / N^{p 1} \\
& P, \frac{\exists X \cdot(X=\operatorname{argmgx}|[Y, P(Y)\}|) \wedge[]\} \mid)}{X}
\end{aligned}
$$

We take predicates to be distributive or collective: in John gave Mary flowers, gave is used in its collective sense for its second argument; in Flowers are beautiful, is beautiful is used in its distributive sense in its first argument.

Cardinal determiners have two built-in meaning components: an existential component and a cardinality one. We propose the following two alternative lexical entries for card, one for the referential (weak) meaning there are card Ps..., the other for the strong meaning there are exactly card Ps...:

$$
\begin{aligned}
& \frac{\frac{S \mid S}{D P Q !}}{N^{P !}} \\
& \text { card } \\
& \lambda P, \frac{\exists X,|X|=\operatorname{card} A P(X) \Lambda[]}{X}
\end{aligned}
$$

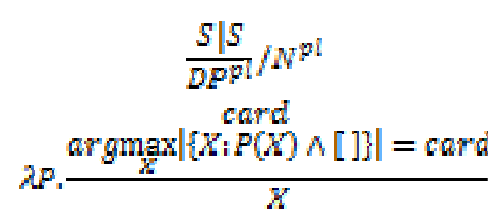

The semantic ambiguity between the two lexical entries of a cardinal card is determined by whether the scope of the following context (continuation) lies inside the scope of the cardinality (as in the second entry) or not (as in the first entry).

Both these weak and strong meaning of cardinal may bind subsequent anaphora (as opposed to the case of some that can bind only with its maximal meaning). For the weak meaning, we can just use the regular Bind rule, whereas for the strong meaning (exactly card), one cannot use Bind because that would bring the continuation into the scope of argmax, altering the truth conditions. Thus, we have to force the scope closing of argmax immediately after the interpretation of the cardinal's minimal clause by applying Lower. To allow the strong meaning of card to bind, we have to give it jet another lexical entry:

$$
\begin{aligned}
& \begin{array}{c}
\frac{S \mid D P^{p l}-S}{D p^{p 1} / N^{p l}} \\
\text { card }
\end{array} \\
& \exists X .[]
\end{aligned}
$$

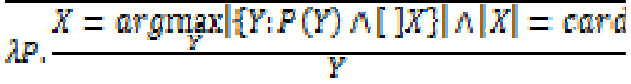

These representations are not completely satisfying because the lexical ambiguity of plural quantificational determiners generates an explosion of ambiguous representation of the discourse in which the determiners are used. We leave the problem of finding a more general solution for a unitar representation of the plural quantificational determiners some, most and cardinals to further research.

\section{Distributive Vs. COLLECTIVE READING}

We will consider two of the most influential existing strategies to deal with plurals and their associated ambiguities (collective, distributive or cumulative readings): Scha [17] and Link [13]. Scha and Link locate the source for the ambiguity of plural sentences differently. According to Scha the ambiguity between collective, distributive and possibly other readings is located in the plural noun phrase or more precisely in the determiner. According to Link, noun phrases are unambiguous and the readings should be generated within the verb phrase. A third strategy proposes that readings of complex sentences are a result of the whole structure or as Roberts [16] puts it: "Distributivity is a property of predications, combinations of a subject and a predicate."The readings can be triggered by different elements of a sentence; there is a functional interplay between the different categories."

We will take predicates, not nouns to be distributive, collective, or ambiguous. We will not commit ourselves to whether the distributivity comes as a feature from the lexical semantics, or it is entailed from the world knowledge and the sense of the predicate itself [16]. Here are some examples: 
Sue and Mary are pregnant. (be pregnant is a distributive predicate)

John and Bill moved the piano. (moved is an ambiguous between distributive and collective predicate)

The students gathered in the square. (gathered is a collective predicate)

As a general rule, we posit that a distributive predicate $\mathrm{P}_{\text {dist }}$ is true of a plural referring variable $\mathrm{X}=\left\{\mathrm{x}_{1}, \mathrm{x}_{2}, \ldots \mathrm{x}_{\mathrm{n}}\right\}$ iff $\mathrm{P}_{\text {dist }}\left(\mathrm{x}_{1}\right) \wedge \mathrm{P}_{\text {dist }}\left(\mathrm{x}_{2}\right) \wedge \ldots \wedge \mathrm{P}_{\text {dist }}\left(\mathrm{x}_{\mathrm{n}}\right)$. And a collective predicate $P_{\text {call }}$ is true of a plural referring variable $X=\left\{x_{1}, x_{2}, \ldots x_{n}\right\}$ iff $\mathrm{P}_{\text {call }}\left(x_{1} \wedge \mathrm{x}_{2} \ldots \wedge \mathrm{x}_{n 2}\right)$. Note that a predicate may have multiple arguments (subject and direct object, for instance). So a predicate may be distributive or collective in each of the arguments.

\section{COORDONATION: CONJUNCITON AND DISJUNCTION}

The work [2] gives the following lexical entry for or:

$$
\begin{gathered}
\left(A \backslash \frac{S \| S}{A}\right) / A \\
\partial \operatorname{or}\left(\lambda \lambda L \frac{(\lambda, k(L) \vee k(R))\left(\lambda x_{1}[]\right)}{x}\right.
\end{gathered}
$$

The lexical entry for or is polymorphic: $A$ can be any category, such as $D P, \quad D P \mid S$ (verb phrases), $D P \mid D P$ (adjectives) or $S$ (sentence). Partee and Rooth [15] are the first to suggest allowing phrases like John or Bill to introduce new variables.

We point that disjunction may introduce only singular variables:

John owns a donkey or a goat. He beats it/* them.

John or Bill called. He/*They hang up.

We straightforwardly extend the semantic representation for disjunction from [2] to conjunction:

$$
\begin{gathered}
\left(A \backslash \frac{S \mid S}{A}\right) / A \\
\text { and } \\
\lambda \operatorname{RaL} \cdot \frac{(\lambda k \cdot k(L) \wedge k(R))(\lambda x .[])}{x}
\end{gathered}
$$

Note that and is also polymorphic. Thus it may account for discourses like: John drinks and talks. He does this for hours, where this is anaphoric to plural events, provided only we modify the binding rule to allow categories other then $D P$ (like $D P(S)$ to bind subsequent pronouns. Note also that conjoined $D P$ s have the power to introduce variables that may be further referred by plural pronouns, a power disjoint $D P$ s do not have:

John owns a donkey and a goat. He beats *it/ them. John and Bill called. *He/They hang up.

\section{PluRAl PRONOMINAL ANAPHORA}

Ideally, singular and plural pronominal anaphora should behave similarly and be parallel phenomena. Unfortunately, at a closer look, there are striking differences between the anaphoric properties of singular and plural pronouns. On the one hand, only singular $D P$ s have the power to introduce singular variables that could bind subsequent singular pronominal anaphora. On the other, plural variables may be introduced not only by plural $D P \mathrm{~s}$, but also by: two or more singular DPs, coordinated (John and Mary came. They whistled) or not (John took Mary to Acapulco. They had a lousy time), or by quantificational singular DPs (Every man came. They whistle or A kid climbed every tree. They were full of energy).

We first treat the simplest case, that of plural entities introduction by plural $D P$ s (analogous to singular entity introduction). Plural $D P$ s are formed of plural quantificational determiners such as some, all or most and a plural common noun required as argument by the determiner. We take singular common nouns to be functions (properties) of individual variables $x$, while plural common nouns expect a plural individual variable $X$. Thus, for such (non-specific) antecedents of they, we may use the following lexical entry:

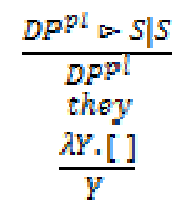

Here is the derivation for Some kids came. They played:

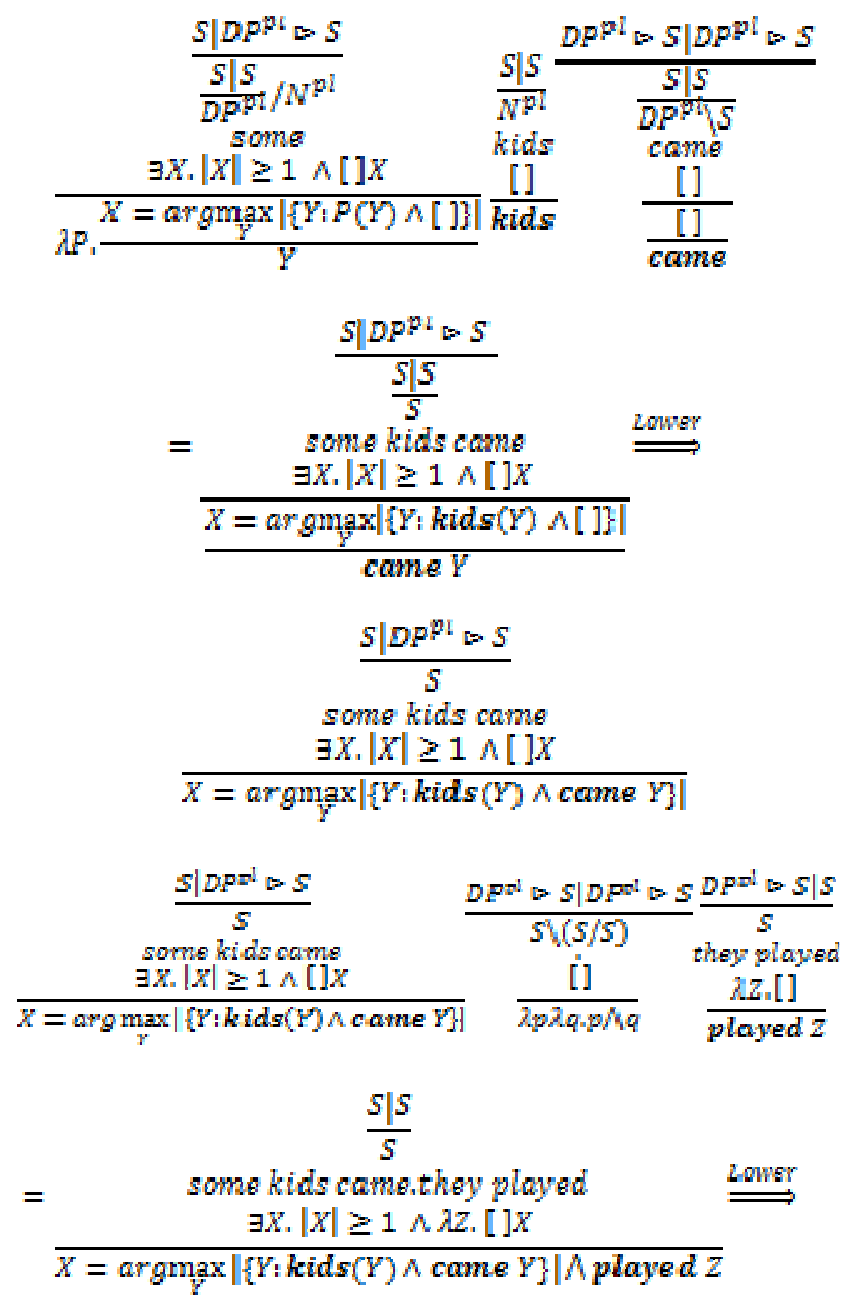




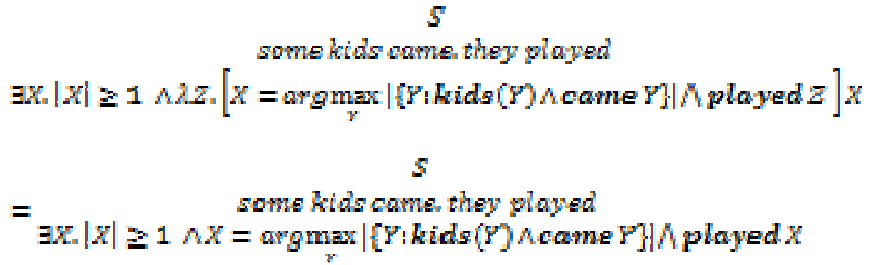

which amounts to saying that there is a plural entity $X$ of cardinality at least one, formed of all the kids that came and that plural entity $X$ played. In a similar manner one obtains the derivation for Most kids came. They played and for All kids came. They played.

As for the plural anaphora introduced by cardinal determiners, consider the following two examples: Five men walk in the park. They watch the birds. (preferred reading: there are some context relevant five men and they walk in the park and they watch the birds; there could be other not contextually important men walking and watching); Five men walk in the park and watch the birds. (preferred reading: there are exactly five man who are in the park and watch the birds). We take both examples to be semantically ambiguous between two readings which correspond to the two scope-distinct lexical entries for the cardinal determiner five. Pragmatic reasons dictate the preferred reading in each case. We give the interpretations of these preferred readings (and skip the not preferred ones, though semantically possible), ignoring the full interpretation of walk in the park and of watch the birds:

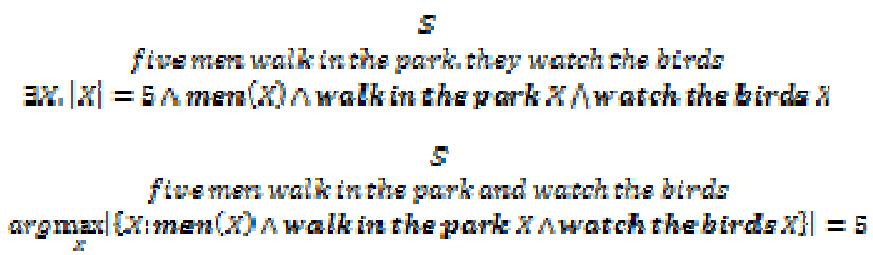

We turn now to the case of introducing plural entities by coordination (conjunction or disjunction). The lexical entry for conjunction obviously gives right truth conditions and offers an antecedent for subsequent anaphora, as in, for example: John and Mary came. They whistled. In such cases, where more than one specific antecedent is present in the discourse, the lexical entry for they needs to search left for two (or three, or another number) $D P$ s, for instance:
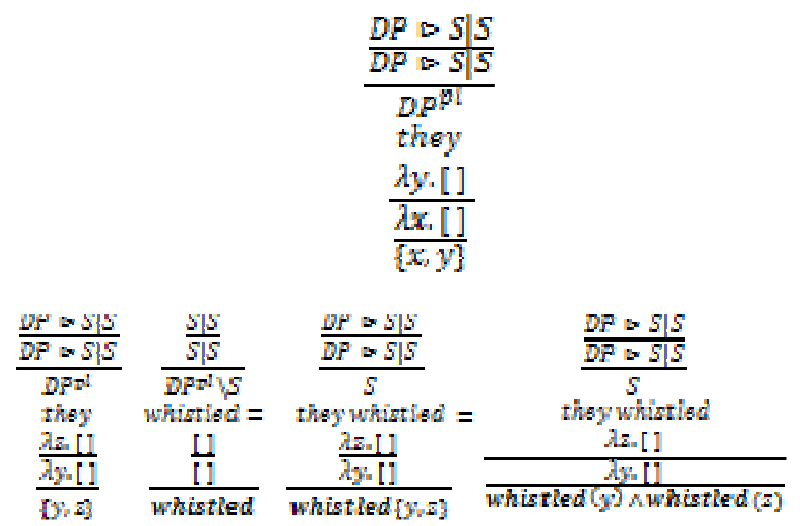


\section{REFERENCES}

[1] C. Barker and Chung-chieh Shan, "Donkey anaphora is in-scope binding," Semantics \& Pragmatics, Volume 1, pp. 1-46, 2008.

[2] C. Barker, "Continuations and the nature of quantification," Natural Language Semantics, 10(3). 211-242, 2002.

[3] C. Barker, "Continuations in natural language," in Proceedings of the fourth ACM SIGPLAN workshop on continuations, Hayo Thielecke (ed.), pp. 55-64, 2004.

[4] A. Dinu, "Versatility of continuations in discourse semantics," Fundamenta Informaticae, 18 pp., 2011.

[5] G. Evans, "Pronouns, Quantifiers and Relative Clauses I \& II," Canadian Journal of Philosophy, 7, 1977.

[6] M. Felleisen, "The theory and practice of first-class prompts," in Proceedings of the Fifteenth Annual ACM Symposium on Principles of Programming Languages, J. Ferrante and P. Mager (eds), p. 180-190, San Diego, California, ACM Press, 1988.

[7] J. A.G. Groenendijk and M.B.J. Stokhof, "Dynamic Montague Grammar," in Papers from the Second Symposium on Logic and Language, L. Kalman and L. Polos (eds.), Budapest: Akadémiai Kiadó, 1990.

[8] J. Groenendijk and M. Stokhof, "Dynamic predicate logic," Linguistics and Philosophy, 14(1) 39-100, 1991.

[9] P. de Groote, "Towards a Montagovian account of dynamics," Semantics and Linguistic Theory XVI, 2006.

[10] I. Heim, "File change semantics and the familiarity theory of definiteness," in Meaning, Use and the Interpretation of Language, Rainer Bauerle, Cristoph Schwarze, and Arnim von Stechow (eds), Walter de Gruyter \& Co., 1983.

[11] P. Jacobson, "Towards a variable-free semantics," Linguistics and Philosophy, 22(2). 117-185, 1999.

[12] H. Kamp and U. Reyle, From Discourse to Logic, Kluwer Academic Publishers, 1993.

[13] Link, G.: The logical analysis of plurals and mass terms: A latticetheoretical approach. In Meaning, use and the interpretation of language, eds. R. Bäuerle, C. Schwarze and A. von Stechow, 303-323. Berlin, New York: Walter de Gruyter, 1983.

[14] R. Montague, "The Proper Treatment of Quantification in English," in Formal Philosophy: Selected Papers of Richard Montague, R. Thomason (ed.), pp. 247-270. New Haven:Yale, 1970.

[15] B. H. Partee and M. Rooth, "Generalized conjunction and type ambiguity," in Meaning, use and interpretation of language, Rainer Bäuerle, C. Schwarze, and A. von Stechow (eds.), pp. 361-383, de Gruyter, 1983.

[16] C. Roberts, Modal subordination, anaphora and distributivity. $\mathrm{PhD}$ dissertation, UMass. Amherst, 1987.

[17] R. Scha, "Distributive, collective and cumulative quantification," in Formal Methods in the Study of Language, J. Groenendijk, M. Stokhof, and T.M.V. Janssen (eds.), Mathematisch Centrum, Amsterdam, 1981.

[18] Chung-chieh Shan and C. Barker, "Explaining crossover and superiority as left-to-right evaluation," Linguistics and Philosophy, 29.1:91-134, 2006.

[19] Chung-chieh Shan, Linguistic side effects, Ph.D. thesis, Harvard University, 2005. 